\title{
ASPECTOS GENERALES PARA LA HABILITACIÓN DE UNA FARMACIA DE PREPARACIONES
} EN COSTA RICA

Recibido: 28-07-18

Aceptado: 06-08-18
POR: César Andrés Barrantes Murillo1

1 Autor del artículo cesbar27@hotmail.com

Estudiante de Farmacia, Curso de Farmacotecnia, Universidad de Ciencias Médicas (UCIMED)

Dr. Luis Carlos Monge Bogantes 2

2 Farmacéutico y Docente Universitario, Curso de Farmacotecnia, Universidad de Ciencias Médicas (UCIMED)

\section{Resumen}

Las preparaciones magistrales, como cualquier otra práctica farmacéutica, demandan el cumplimiento de una serie de requisitos mínimos de calidad, eficacia y seguridad, los cuales son regulados mediante las legislaciones adoptadas por cada país. Actualmente Costa Rica carece de una estructura sólida para el control de establecimientos de este tipo, lo cual ha desencadenado la necesidad de modificar el sistema empleado y emular los de países como España, Argentina y Chile, en donde la regulación ha sido optimizada.

Palabras Clave: preparaciones magistrales, control de calidad, medicamentos, farmacia

\section{Abstract}

Magisterial preparations, like any other pharmaceutical practice, demand the fulfillment of a series of minimum requirements of quality, efficacy and safety, which are regulated by the legislations adopted by each country. Currently Costa Rica lacks a solid structure for the control of establishments of this type, which has triggered the need to modify the system used and emulate those of countries such as Spain, Argentina and Chile, where regulation has been optimized.

Keywords: magisterial preparations, quality control, medicines, pharmacy

\section{Antecedentes}

os fármacos producidos a nivel industrial son diseñados en función de englobar las necesidades de toda la población, no obstante, a raíz de las particularidades de los individuos no siempre es posible cubrir los requerimientos especiales de cada paciente. A partir de la problemática señalada anteriormente surge la necesidad de que los profesionales de la salud, específicamente farmacéuticos, diseñen, preparen y dispensen medicamentos magistrales.

El Colegio de Farmacéuticos de la provincia de Buenos Aires (2012) define una preparación magistral como:

"Todo medicamento prescrito en una receta magistral para un paciente individualizado, poste riormente preparado, envasado y rotulado por un Farmacéutico en el laboratorio de su Farmacia y dis 7 pensado en la misma, para cumplir expresamente una prescripción facultativa detallada de los principios activos que incluye" (Colegio de Farmacéuticos de Buenos Aires, Argentina; 2012, p 13).

Cabe destacar que al igual que los fármacos industrializados y cualquier otro producto relacionado con el área de la salud (por ejemplo dispositivos médicos, medicamentos o dermocosméticos, entre otros), las preparaciones magistrales requieren un sistema regido por las Buenas Prácticas, el cual garantice la seguridad del paciente y una actividad terapéutica eficaz. A partir de lo anterior nacen una serie de requisitos mínimos los cuales deben de cumplir los establecimientos farmacéuticos de este tipo; el presente artículo busca presentar desde un punto de vista crítico, las legislaciones y reglamentos implementados actualmente en Costa Rica referentes a la apertura de una farmacia de preparaciones magistrales y llevar a cabo un análisis 
comparativo, en el cual se pueda contrastar la situación vigente con los sistemas adoptados por otros países en el mundo.

\section{Situación actual en Costa Rica}

En el caso de Costa Rica actualmente, los establecimientos farmacéuticos privados (farmacias comunales privadas) de preparaciones magistrales, se pueden dividir en 2 grupos principales, el primero de ellos a menor escala, como por ejemplo las áreas de preparaciones magistrales en farmacias. Para la habilitación de una Farmacia de Preparaciones el Colegio de Farmacéuticos y el Ministerio de Salud, establecen como requisito apegarse Manual de Normas para la Habilitación de Farmacias Decreto No. 31969-S el cual rige las condiciones y requerimientos mínimos que deben cumplir las farmacias encargadas de dispensar medicamentos. El segundo tipo de establecimiento farmacéutico es aquel en el cual, aunque se llevan a cabo preparaciones magistrales, se realizan a mayor escala (un nivel más industrial), en este caso a nivel nacional se exige cumplir, mientras aplique, con lo establecido en el Reglamento Técnico Centroaméricano TCA 11.03.42:07: Productos Farmacéuticos Medicamentos De Uso Humano, Buenas Prácticas de Manufactura para la Industria Farmacéutica.

Aunque como ya se mencionó, sí existe algún tipo de regulación, se debe hacer énfasis que el material con el cual se manejan los requerimientos de apertura en Costa Rica es deficiente, esto a raíz de que los decretos y reglamentos

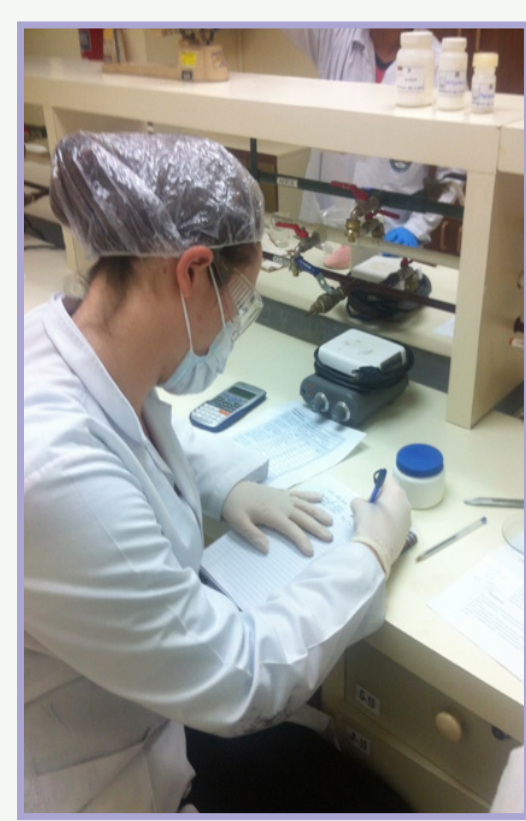

utilizados no logran cubrir todos los aspectos necesarios para asegurar preparaciones magistrales seguras y eficaces.

\section{El Manual de Normas} para la Habilitación de Farmacias Decreto No. 31969-S es un documento en el cual se establece todo aquello que debe de tener una farmacia ya sea pública, privada o mixta para poder desempeñarse de forma legal en el país; entre tantos puntos que se manejan, en él se hace referencia a lo que se necesita para poder contar con un área de preparación magistral de medicamentos.

El problema con el decreto anteriormente mencionado radica en que los requisitos solicitados para dicha "sección" del establecimiento son prácticamente nulos, en las diez páginas de extensión del manual, se hace referencia concretamente en dos ocasiones a un área de magistrales en donde se menciona que esta debe de ser un cubículo aislado y contar con un equipo mínimo para dicha práctica (espátula, mortero, pistilo, probeta, goteros, balanza y pesas granatarias). Esencialmente esto significa que en este país cualquier farmacia puede fabricar medicamentos magistrales sin necesidad de preocuparse por Buenas Prácticas de Manufactura, Almacenamiento y Laboratorio y aun así ser considerada "legal".

En el caso de establecimientos de preparaciones a mayor escala, la situación no mejora; aunque si bien es cierto el Reglamento Técnico Centroaméricano TCA 11.03.42:07: Productos Farmacéuticos Medicamentos De Uso Humano, Buenas Prácticas de Manufactura para la Industria Farmacéutica logra englobar una cantidad muy superior de requerimientos a nivel de estructura, laboratorio, higiene y seguridad sigue teniendo un enfoque excesivamente general hacia la industria, con lo que deja de lado todas aquellas necesidades intrínsecas de un establecimiento farmacéutico enfocado a la elaboración de medicamentos magistrales, y al seguimiento farmacoterapéutico adecuado para con los pacientes, tema del que tan siquiera se hace mención, en dicho reglamento.

\section{Contraste con el ámbito internacional}

Para reforzar la idea planteada anteriormente, basta con analizar los sistemas adoptados por otros países, en el caso de Argentina y específicamente la provincia de Buenos Aires se maneja una Norma de carácter obligatorio, emitida por el Colegio de Farmacéuticos de dicha ciudad llamada "Buenas Prácticas de Preparación en Farmacia". Este documento cubre, entre tantos aspectos de las preparaciones magistrales, elementos como condiciones e infraestructura específica para cada tipo de laboratorio (preparaciones de comprimidos, homeopatía o estériles), equipos y mantenimiento, documentación de procesos 
(ingreso de materia prima, preparación de productos, registros de medicamentos, entre otros), materia prima y envases, estudios de formulación, etiquetado, proceso de dispensación y farmacovigilancia

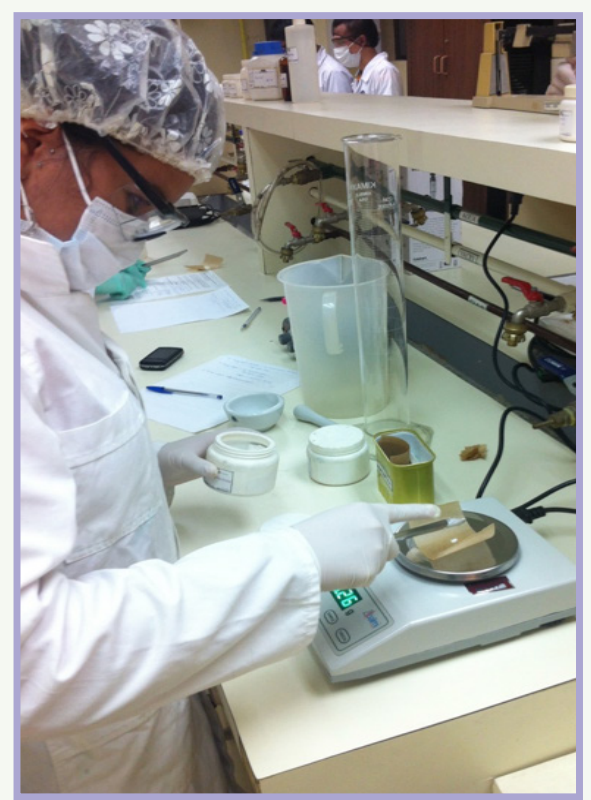

Se debe hace un énfasis a que dicha norma implementa mucho la temática de trazabilidad, la cual permite registrar a d e c u a d a m e n t e la historia de cada preparado. De forma muy similar en Chile el Instituto de Salud Pública desarrolló un documento denominado como "Normas de Buenas Prácticas de Elaboración y Control de Calidad de Fórmulas Magistrales y Preparados Oficinales" el cual llegó a solucionar la falta de disposiciones específicas para aquellas preparaciones elaboradas en Farmacias.

En el caso de España se posee el Real Decreto 175/2001 en donde se aprobó una normativa para regir la elaboración fórmulas magistrales, al igual que en los dos ejemplos anteriores esta logra abarcar todas aquellas condiciones específicas de preparaciones oficinales necesarias para asegurar a los pacientes medicamentos seguros y funcionales; no obstante, lo más destacable del sistema español es que no se queda ahí, sino que también manejan una "Guía de Buenas Prácticas de Preparación de Medicamentos en Servicios de Farmacia Hospitalaria" en la cual no solo se incluyen todos los puntos mencionados anteriormente, sino que también se da un enfoque aún más detallado al área de servicios de farmacotecnia a nivel de hospital propiamente.

En la farmacopea de los Estados Unidos (USP-NF) en la sección con al menos 40 capítulos generales en los que se describe ampliamente las condiciones en las que se deben elaborar las preparaciones magistrales. Además de contar con todo un suplemento de casi 700 páginas denominado Compounding Compendium - USP-NF, actualizado hasta junio del 2018. Se encuentra la monografía de más de 170 preparaciones oficinales.
Tras conocer un poco acerca del manejo que se tiene a nivel regulatorio, en cuanto al tema de preparaciones magistrales, en otros lugares del mundo, realmente deja evidenciada la deficiencia que existe en Costa Rica. Basta con leer normativas como la Argentina para darse cuenta que el "Manual de Normas para la Habilitación de Farmacias Decreto No. 31969-S" deja de lado una cantidad escandalosa de consideraciones fundamentales para que una Farmacia de Preparaciones pueda ser habilitada de forma responsable.

\section{Acciones correctivas}

Antes de poder plantear soluciones es fundamental entender el origen del problema, Costa Rica como se ha podido apreciar a lo largo de este artículo es un país en el cual se ha dejado de lado a las preparaciones magistrales como rama de la profesión farmacéutica. Mi precepción como estudiante es que en las últimas décadas ha predominado la mentalidad de que todos los requerimientos de la población se encuentran cubiertos por la producción industrial, por lo que se ha generado una desvinculación del farmacéutico con dicha práctica y por ende poco interés para avanzar como país en este ámbito. Aunado a lo anterior, lamentablemente la formación que tienen los profesionales en Costa Rica acerca del tema es prácticamente nula, lo cual de cierta forma explica porque existe un subdesarrollo tan marcado con respecto a las demás naciones.

Para poder corregir
de forma certera la
problemática expuesta
en este artículo se deben
de tomar una serie de
medidas inmediatas,
personalmente considero
que estas podrían
subdividirse en tres
enfoques específicos.
El primero de ellos es
quizá el más lógico,
básicamente sería
plantearse la posibilidad
de modificar las
normativas y reglamentos


que rigen a los establecimientos de preparaciones en este país, idealmente generando legislaciones específicas para ellos.

Una vez completada la primera etapa se daría paso a la segunda acción correctiva, la cual en síntesis sería basar las modificaciones del sistema actual, en las implementadas por otros países, está más que claro que por el mismo estancamiento de Costa Rica en el área no es viable tratar de abarcar todo los aspectos cubiertos en el extranjero, no obstante, lo ideal sería iniciar con los elementos más críticos en términos de calidad para los productos magistrales y con el tiempo ir aumentando el alcance de manera que adoptemos una ideología de mejora continua.

El último enfoque es más desligado a los dos primeros y va direccionado principalmente al origen del problema, la cultura y educación del farmacéutico promedio en Costa Rica. Como ya se mencionó, la falta de compromiso o el hecho de que las preparaciones magistrales no sean una prioridad en el ejercicio profesional de la farmacia han desencadenado el abandono de dicha práctica, hasta el punto que carecemos de una legislación seria que la regule; a raíz de lo anterior creo necesario un cambio a nivel cultural, tanto para profesionales ya graduados como para estudiantes de la carrera.

Para los primeros la situación es un poco más compleja, debido a que no todas las personas comparten ese compromiso hacia el aprendizaje continuo, sin embargo, el uso de campañas informativas, talleres y conferencias para las personas colegiadas del país podría marcar la diferencia; por otra parte, el tema de los estudiantes representa una ventana infinita de oportunidades, ya que este sector es más manejable y receptivo, el simple hecho de incluir a las preparaciones magistrales como requisito obligatorio en la formación de farmacéuticos puede maximizar el alcance de los futuros profesionales, de manera que el progreso será más efectivo.

\section{Conclusión}

Como resultado de la investigación realizada, es posible concluir que Costa Rica requiere de un cambio en su estructura, percepción, formación y regulación hacia los laboratorios de preparaciones magistrales. Si bien es cierto el proceso no es sencillo ni rápido, si se trabaja en función de mejorar el sistema implementado actualmente y generar un impacto a nivel cultural en los farmacéuticos, es totalmente factible pensar en que podríamos seguir e inclusive alcanzar el patrón de trabajo de países como Argentina, Chile o

\section{Referencias Bibliográficas}

- Instituto de Salud Pública. (2006). Normas de Buenas Prácticas de Elaboración y Control de Calidad de Normas Magistrales y Preparados Oficinales. Departamento Control Nacional/ Subdepartamento Químico Analítico. Santiago: ISP.

- Ministerio de Sanidad, Servicios Sociales e Igualdad. (2014). Guía de Buenas Prácticas de Preparación de Medicamentos en Servicios de Farmacia Hospitalaria. España: Dirección General de la Cartera Básica de Servicios del SNS y Farmacia. Obtenido: https://www.sefh.es/sefhpdfs/GuiaBPP_JUNIO_2014_VF.pdf.

- Ministerio de Salud. (2008). Decreto No. 31969-S. Manual de Normas para la Habilitación de Farmacias. Publicado en La Gaceta N ${ }^{\circ}$ 81, del 28 de abril de 2008. Costa Rica.

- Ministerio de Salud de la Provincia de Buenos Aires. (2012). Resolución 8 /12. Buenas Prácticas de Preparación en Farmacia. Publicado en $\neg$ Colegio de Farmacéuticos de la Provincia de Buenos Aires, abril de 2012. Argentina.

- Ministerio de Sanidad y Consumo. (2001). Real Decreto No. 175/2001. Normas de Correcta Elaboración y Control de Calidad de Fórmulas Magistrales y Preparados Oficinales. Publicado en BOE N 65 , del 16 de marzo de 2001. España.

- Organización Mundial de la Salud. (2007). Resolución Na 214-2007. RTCA 11.03.42:07: Productos Farmacéuticos Medicamentos De Uso Humano, Buenas Prácticas de Manufactura para la Industria Farmacéutica. Publicado en COMIECOXLVII, del 11 de diciembre del 2007. Costa Rica.

- Compounding Compendium, USP. Farmacopea de los Estados Unidos y Formulario Nacional (USP 42 / NF 37, 2018).

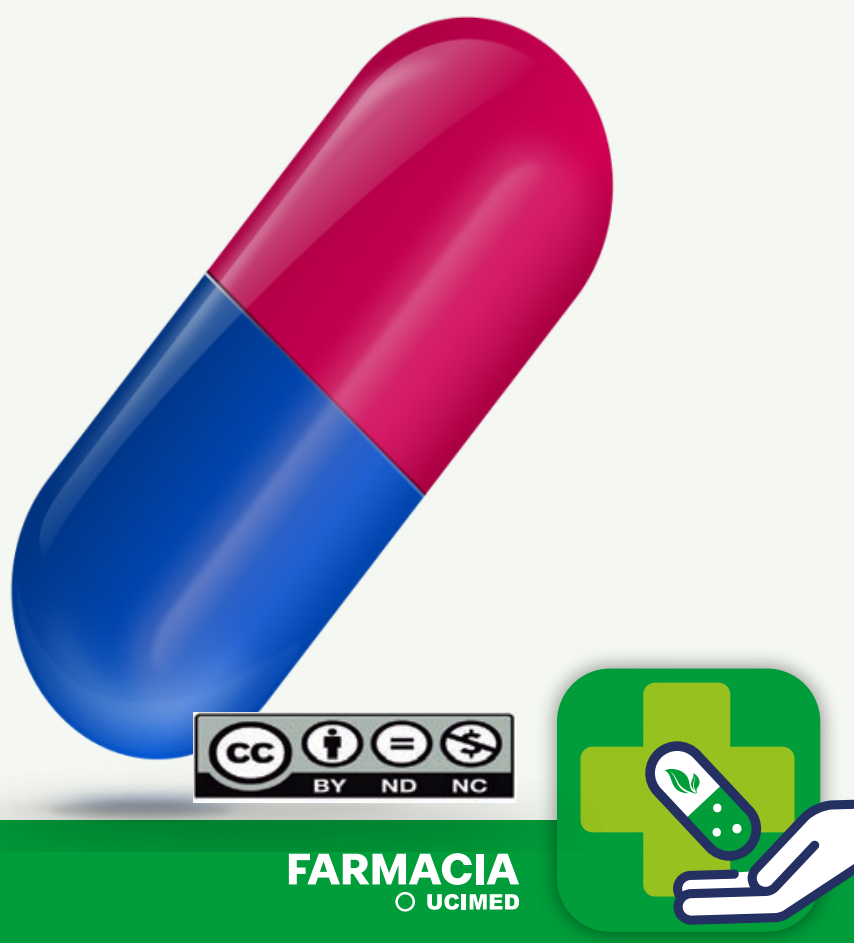

